\title{
Tropospheric dispersive phase anomalies during heavy rain detected by L-band InSAR and their interpretation
}

\author{
Naufal Setiawan ${ }^{1^{*}}$ and Masato Furuya ${ }^{2}$ (i)
}

\begin{abstract}
The split-spectrum method (SSM) can largely isolate and correct for the ionospheric contribution in the L-band interferometric synthetic aperture radar (InSAR). The standard SSM is performed on the assumption of only the first-order ionospheric dispersive effect, which is proportional to the total electron content (TEC). It is also known that during extreme atmospheric events, either originated from the ionosphere or in the troposphere, other dispersive effects do exist and potentially provide new insights into the dynamics of the atmosphere, but there have been few detection reports of such signals by InSAR. We apply L-band InSAR into heavy rain cases and examine the applicability and limitation of the standard SSM. Since no events such as earthquakes to cause surface deformation took place, the non-dispersive component is apparently attributable to the large amount of water vapor associated with heavy rain, whereas there are spotty anomalies in the dispersive component that are closely correlated with the heavy rain area. The ionosonde and Global Navigation Satellite System (GNSS) rate of total electron content index (ROTI) map both show little anomalies during the heavy rain, which suggests few ionospheric disturbances. Therefore, we interpret that the spotty anomalies in the dispersive component of the standard SSM during heavy rain are originated in the troposphere. While we can consider two physical mechanisms, one is runaway electron avalanche and the other is the dispersive effect due to rain, comparison with the observations from the ground-based lightning detection network and rain gauge data, we conclude that the rain dispersive effect is spatiotemporally favorable. We further propose a formulation to examine if another dispersive phase than the first-order TEC effect is present and apply it to the heavy rain cases as well as two extreme ionospheric sporadic-E events. Our formulation successfully isolates the presence of another dispersive phase during heavy rain that is in positive correlation with the local rain rate. In comparison with other dispersive phases during Sporadic-E episodes, the dispersive heavy rain phases seem to have the same order of magnitude with the ionospheric higher order effects.
\end{abstract}

Keywords: Heavy rain, Non-dispersive medium, Dispersive medium, Interferometric synthetic aperture radar, Splitspectrum method

\section{Introduction}

InSAR is one of the space geodetic techniques that can map ground displacements with unprecedented spatial resolution without installing ground-based stations like

\footnotetext{
*Correspondence: naufal.setiawan89@gmail.com

${ }^{1}$ Department of Natural History Sciences, Graduate School of Science,

Hokkaido University, Sapporo, Japan

Full list of author information is available at the end of the article
}

GNSS, which is made possible by processing two images of Synthetic Aperture Radar (SAR) at the same area captured at different times (e.g., Hanssen 2001). Both InSAR and GNSS, are based on phase measurement of the carrier microwaves, which experiences either a delay or advance, depending on the propagation media that are different than vacuum (e.g., Hanssen 2001; Hobiger and Jakowski 2017). The delay or advance is caused by the refractivity of the propagating medium, which is

\section{Springer Open}

(c) The Author(s) 2021. Open Access This article is licensed under a Creative Commons Attribution 4.0 International License, which permits use, sharing, adaptation, distribution and reproduction in any medium or format, as long as you give appropriate credit to the original author(s) and the source, provide a link to the Creative Commons licence, and indicate if changes were made. The images or other third party material in this article are included in the article's Creative Commons licence, unless indicated otherwise in a credit line to the material. If material is not included in the article's Creative Commons licence and your intended use is not permitted by statutory regulation or exceeds the permitted use, you will need to obtain permission directly from the copyright holder. To view a copy of this licence, visit http://creativecommons.org/licenses/by/4.0/. 
grouped into either dispersive or non-dispersive media. The refractivity in dispersive media depends on the carrier frequency of the microwave, and the ionosphere is the most significant dispersive medium for space geodesy and microwave remote sensing. On the other hand, the refractivity in the troposphere has been modeled as a function of air temperature, surface air pressure, and water vapor pressure (Thayer 1974; Bevis et al. 1994) and is considered to be independent of the carrier frequency particularly in space geodetic techniques. Taking advantage of these differences between ionosphere and troposphere, GNSS operationally utilizes dual-frequency observations not only to estimate the ionospheric TEC (e.g., Saito et al. 1998) but also to separate the water vapor content, thereby playing a role of atmospheric sensing tool as well (e.g., Bevis et al. 1994; Shoji 2013).

Meanwhile, even in L-band InSAR, no operational dual-frequency analysis has been done until recently as it is based only on a single carrier-frequency observation. Therefore, several approaches have been proposed, such as range split spectrum (Brcic et al. 2010; Rosen et al. 2010; Gomba et al. 2016), range phase group delay (Meyer et al. 2006), and multiple aperture interferometry and its along-track integration (Jung et al. 2013). One recently well-accepted technique is SSM that takes advantage of its finite bandwidth due to the linear frequency modulation in the original radar pulse (Brcic et al. 2010; Rosen et al. 2010; Gomba et al. 2016). To enhance the range resolution in SAR imageries, instead of transmitting a short and intense pulse, each radar pulse is frequency-modulated, so that instantaneous frequency will change linearly over the pulse length (Curlander and McDonough 1991; Hanssen 2001); the wider the bandwidth in the frequency modulation, the better the range resolution. In the SSM, we split the original bandwidth in the range directly into high and low subbands and demodulates into the new higher and lower carrier frequencies (Brcic et al. 2010; Rosen et al. 2010; Gomba et al. 2016; Furuya et al. 2017). Thus, we can derive new InSAR images $\Delta \phi_{\mathrm{H}}$ and $\Delta \phi_{\mathrm{L}}$ and virtually perform dual-frequency InSAR observations, whereas the original range resolution is lost and the proximity of new frequencies might be a problem; the subscript $\mathrm{H}$ and $\mathrm{L}$ stand for higher and lower carrier frequencies. Using the new InSAR images $\Delta \phi_{\mathrm{H}}$ and $\Delta \phi_{\mathrm{L}}$, we can retrieve the dispersive and non-dispersive phase (Gomba et al. 2016):

$$
\begin{gathered}
\Delta \phi_{\text {non-dispersive }}=\frac{f_{0}}{\left(f_{\mathrm{H}}^{2}-f_{\mathrm{L}}^{2}\right)}\left(\Delta \phi_{\mathrm{H}} f_{\mathrm{H}}-\Delta \phi_{\mathrm{L}} f_{\mathrm{L}}\right) \\
\Delta \phi_{\text {dispersive }}=\frac{f_{\mathrm{H}} f_{\mathrm{L}}}{f_{0}\left(f_{\mathrm{H}}^{2}-f_{\mathrm{L}}^{2}\right)}\left(\Delta \phi_{\mathrm{L}} f_{\mathrm{H}}-\Delta \phi_{\mathrm{H}} f_{\mathrm{L}}\right)
\end{gathered}
$$

where $f_{0}, f_{\mathrm{H}}$, and $f_{\mathrm{L}}$ are the original, new higher, and lower carrier frequency, respectively. As noted above, $\Delta \phi_{\text {dispersive }}$ is the contribution of the phase advance due to TEC in the ionosphere, whereas $\Delta \phi_{\text {non-dispersive }}$ includes the effects due to surface deformation and tropospheric delay after a removal of orbital and topographic phases.

The above formula is developed based on the dispersive nature of the ionosphere that follows the Appleton-Hartree equation (Belcher. 2008). Yet, it only accounts for the first-order term in the refractive index that is proportional to the ionospheric TEC and inversely proportional to the frequency-squared, since the higher order terms of the ionosphere is assumed to be negligible as follows:

$$
n=1-40.3 \mathrm{TEC} / f^{2}
$$

However, if extreme ionospheric events occurred, such as Sporadic-E, we may have to consider higher order ionosphere effects as have been considered in GNSS (Kedar et al. 2003) and InSAR (Furuya et al. 2017).

Although no tropospheric dispersive effects are included in Eq. (2), which is generally a good approximation, the refractive index of the troposphere can also depend on the frequency and even become complex (e.g., van de Hulst 1957; Crane 1967; Zufferey 1972; Liebe 1989; Solheim et al. 1999); we assume that the contributions to the imaginary part of refractive index are negligible in L-band. Even though the effect of molecular resonances is negligible for L-band because of its far location from the resonant frequencies, the dispersive effect is caused by forward scattering from nongaseous larger particles ( $>1 \mathrm{~mm}$ in size) such as rain droplet, hail and snow (Solheim et al. 1999). Based on the transition matrix approach for computing scattering properties (Oguchi 1983), Solheim et al. (1999) showed that L1 microwave propagation through 1 $\mathrm{km}$ of heavy rain could cause $15 \mathrm{~mm}$ of delay. To our knowledge, however, no observational verifications have been done on the theoretical prediction, using the real GNSS data during heavy rain.

In addition to the forward scattering mechanism from rain droplet, we may conceive another possible mechanism for tropospheric dispersive phase, motivated by the recent development of High-Energy Atmospheric Physics (e.g., Dwyer et al. 2012). It is now well-established that high-energy particles such as $\mathrm{X}$-ray and Gamma ray are radiated in association with thunderclouds and lightning initiation and detected either in situ by aircraft (Kelley et al. 2015) or on the 

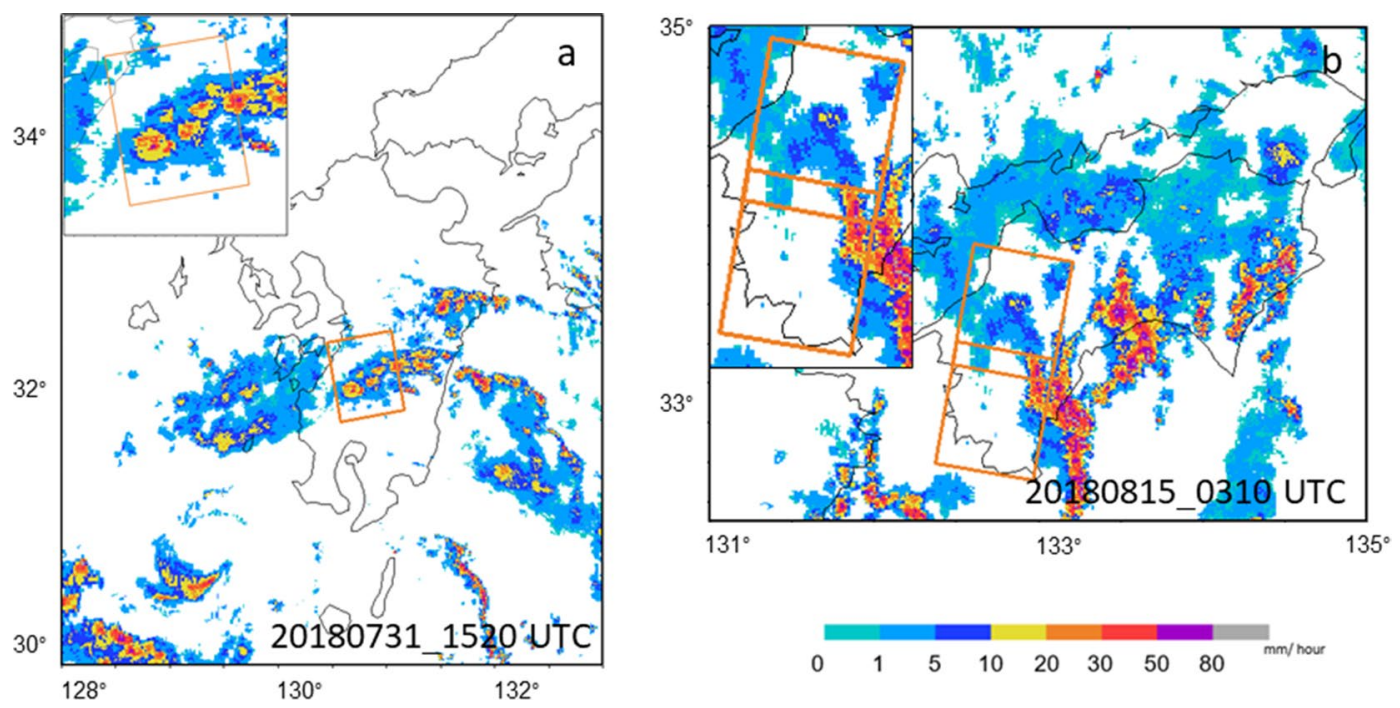

Fig. 1 JMA weather radar observation on a Kyushu at 20180731_1520UTC and b Shikoku at 20180815_0310UTC; we use a convention YYYYMMDD to denote the imaging year, month and date. Orange square denotes the SAR imaging area taken by ALOS-2/PALSAR-2 SM-1 mode

ground (Wada et al. 2018). Those X-ray or Gamma-ray emissions are thought to be caused by bremsstrahlung of the relativistic runaway electron avalanches that are accelerated under the electric field in the troposphere (Gurevich et al. 1992, 2004; Dwyer 2003). Troposphere can thus become natural accelerator and contain accelerated electrons that are, if ever, local and transient, while it is unclear if such electrons could become comparable to the background TEC.

In this study, we first apply the standard SSM to InSAR images acquired during heavy rain (rainfall rate exceeding $50 \mathrm{~mm} / \mathrm{h}$ ) in Japan, so that we can confirm the presence of both huge amount of water vapor in the $\Delta \phi_{\text {non-dispersive }}$ and a long-wave trend due to the ionospheric TEC in the $\Delta \phi_{\text {dispersive; }}$ we confirmed no surface deformation episodes such as earthquakes. In addition to those expected phase anomalies; however, there are spotty anomalies in $\Delta \phi_{\text {dispersive }}$ of the SSM which are clearly neither identical to the long-wavelength ionospheric phase, nor, to the localized strong anomalies due to the Sporadic-E (Furuya et al. 2017).

Then, following two previous physical mechanisms during heavy rain, we examine the spotty anomalies in $\Delta \phi_{\text {dispersive }}$ of the SSM with the ground-based ionosonde, GNSS TEC map, lightning detection network, and rain gauge data. Furthermore, we propose a formulation to examine the presence of another dispersive phase and apply it to heavy rain cases. We attempt to quantify the magnitude of another dispersive phase with rain rate from the weather data by calculating the root mean square (RMS) value. We also test our proposed equation on other extreme atmospheric phenomena that originated in the ionosphere, the Sporadic-E.

\section{Anomaly observed in $\Delta \phi_{\text {dispersive }}$ of SSM during heavy rain \\ Data and processing strategy}

Heavy rain occurrences are detectable using the Japan Meteorological Agency (JMA) weather radar observation

Table 1 Details of InSAR data used in this study

\begin{tabular}{llllll}
\hline Area & Dates (YYYMMDD) & Path frames & $\begin{array}{l}\text { Original, high and low } \\
\text { frequencies (GHz) }\end{array}$ & Split bandwidth (MHz) & $\begin{array}{l}\text { Perpendicular } \\
\text { baseline } \\
\text { (Bperp) }\end{array}$ \\
\hline Kyushu & $20180731-20180828^{\mathrm{a}}$ & $131-630$ & $1.2575,1.2840,1.2310$ & 15.867 & $41.1592 \mathrm{~m}$ \\
& $20180410-20180828^{\mathrm{b}}$ & & & $92.7221 \mathrm{~m}$ \\
Shikoku & $20180815-20181107^{\mathrm{a}}$ & $22-2940,2950$ & $1.2575,1.2840,1.2310$ & $-274.9842 \mathrm{~m}$ \\
& $20180228-20181107^{\mathrm{b}}$ & & & $-337.8612 \mathrm{~m}$ \\
\hline
\end{tabular}

\footnotetext{
${ }^{a}$ Heavy rain
}

${ }^{\mathrm{b}}$ Fair-weather 

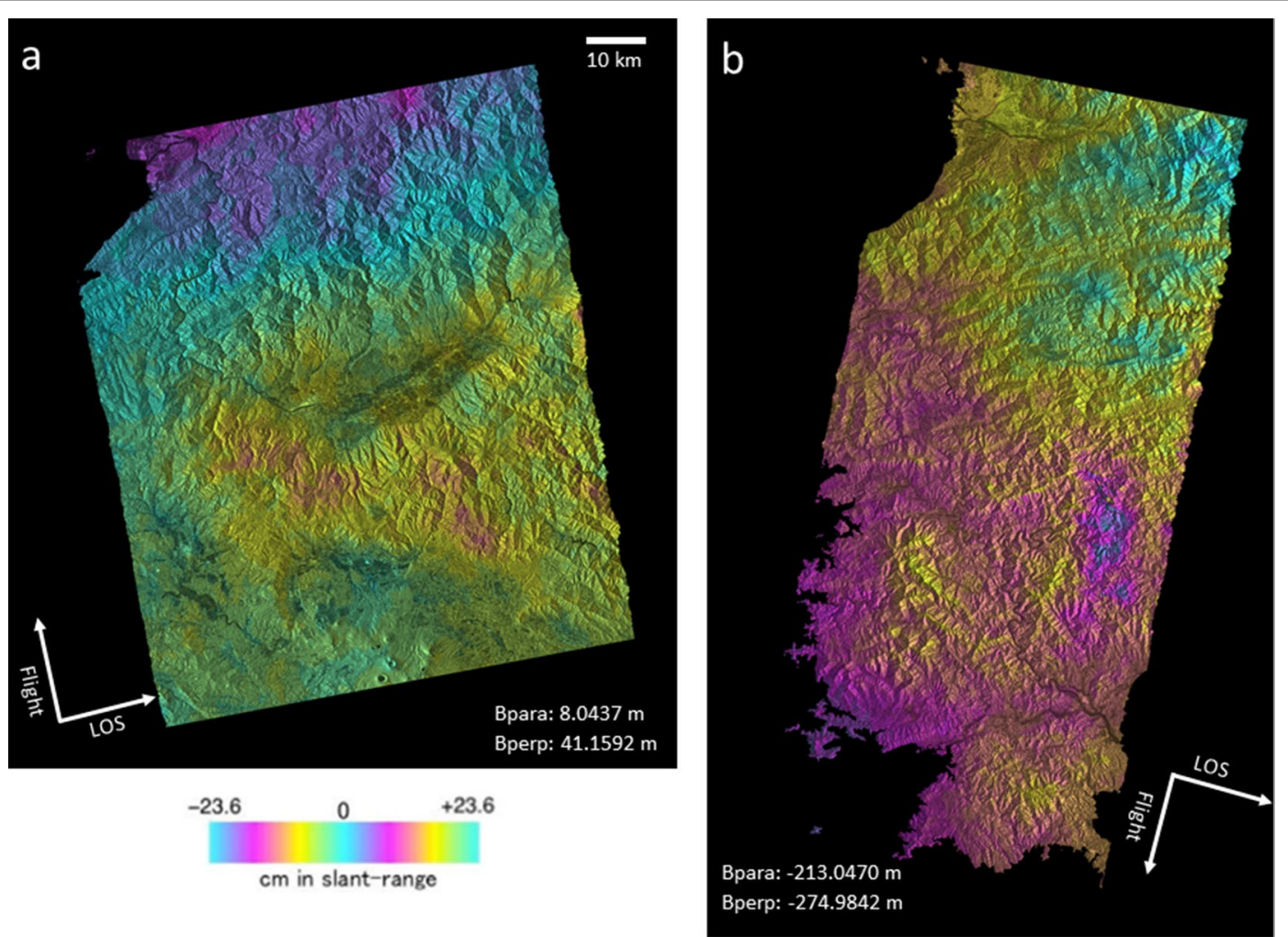

Fig. 2 Original bandwidth differential interferogram of heavy rain pairs. a Kyushu on 20180731_20180828 and b Shikoku 20180815_20181107

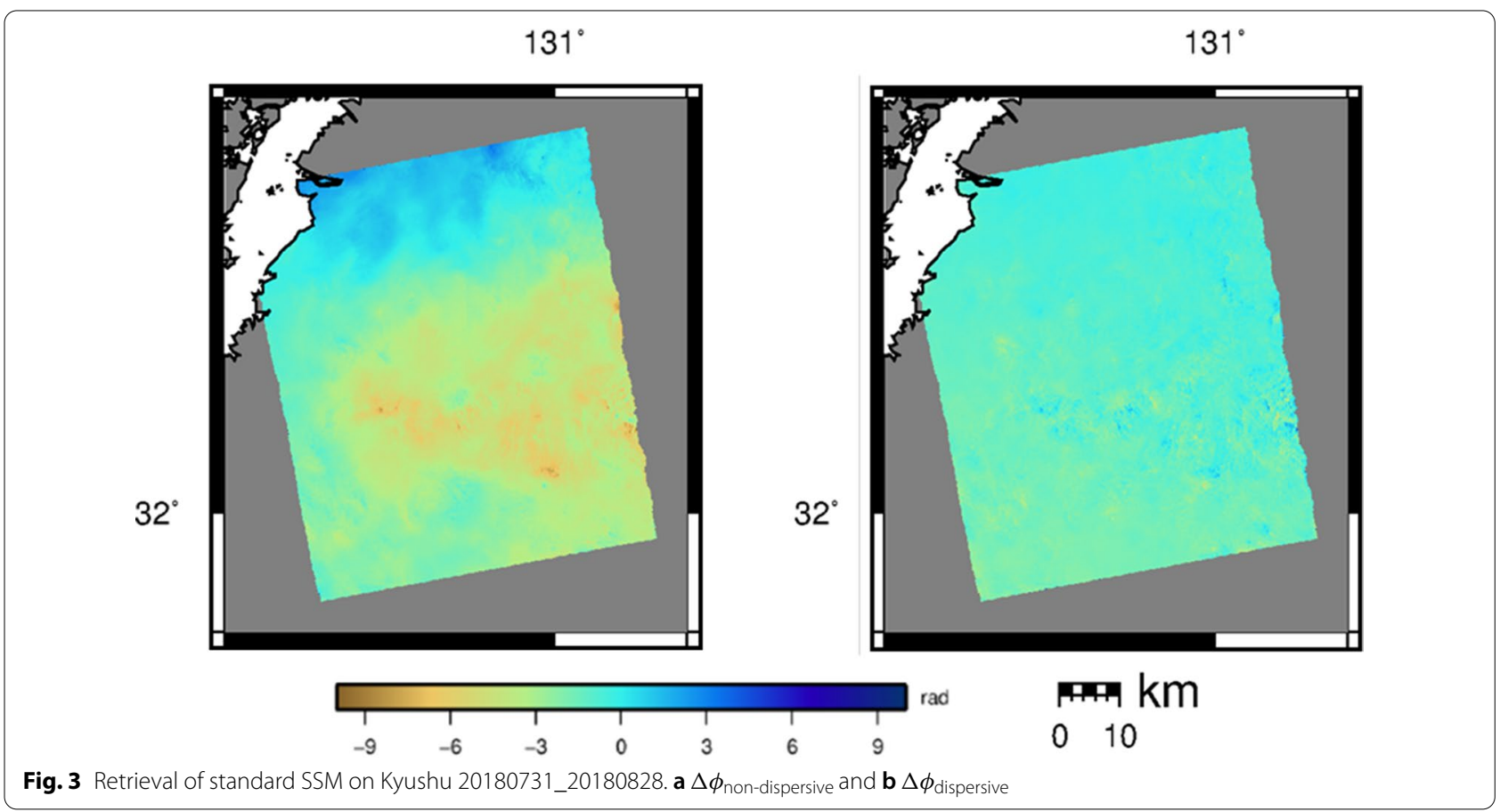




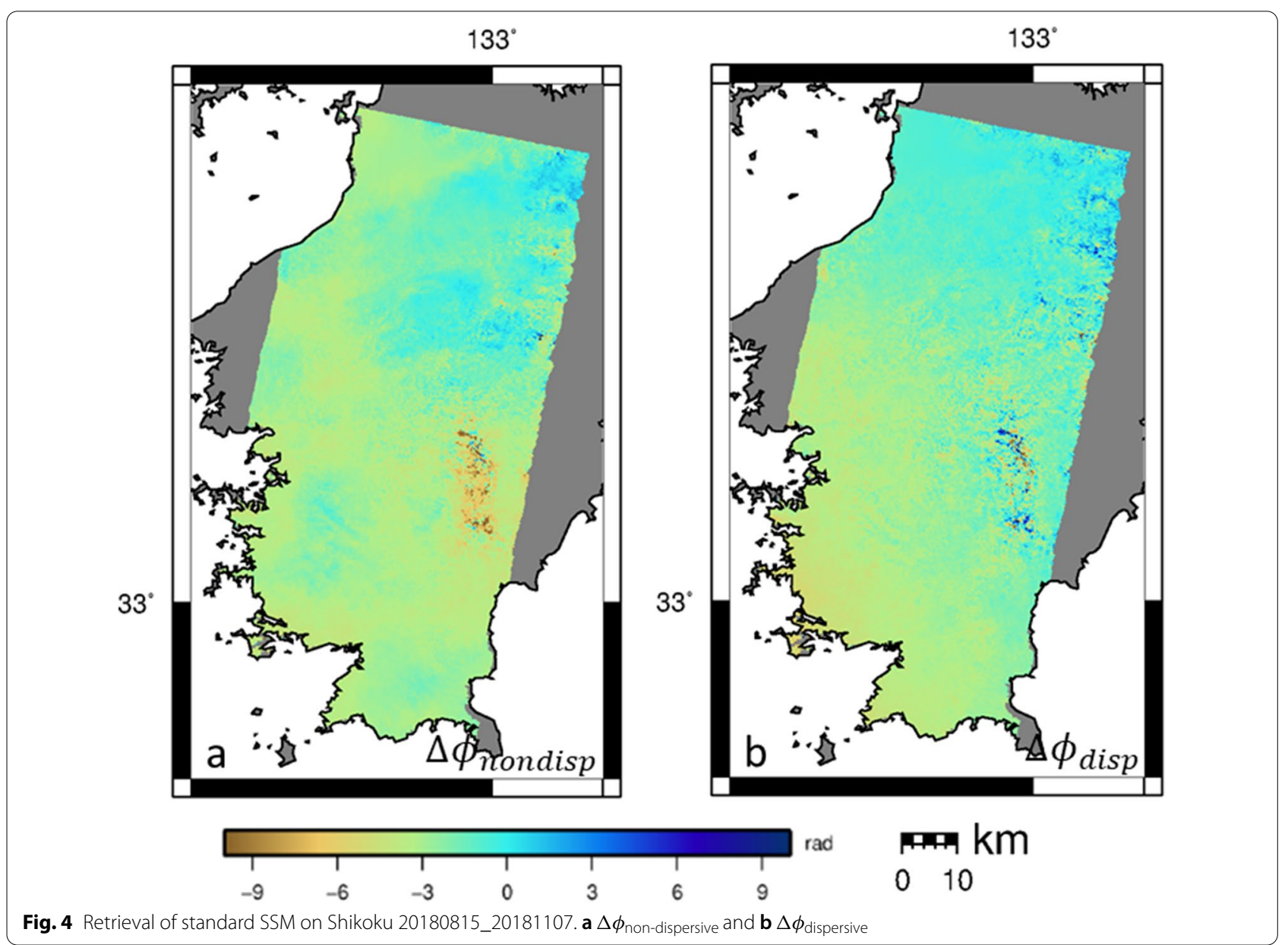

network that consists of 20 rain radar antennas in the whole Japan Island and delivers rain rate every $10 \mathrm{~min}$ over Japan with a spatial resolution of $\sim 1 \mathrm{~km}$ (http:// database.rish.kyoto-u.ac.jp/arch/jmadata/data/jmaradar/synthetic/original/). Then, we look for the data availability for L-band ALOS-2/PALSAR-2 SM-1 mode images; the chirp bandwidth in the SM-1 mode is $79.4 \mathrm{MHz}$. Under the basic observation scenario of ALOS-2/PALSAR-2 over Japanese island, even the shortest recurrent interval for InSAR is 56 days, and thus there are limited opportunities for us to examine the impact of heavy rain in InSAR. Here we report two heavy rain cases in southern Japan, Kyushu on July 31, 2018 (Fig. 1a) and Shikoku on August 15, 2018 (Fig. 1b), respectively. We have confirmed that the other imaging dates were not affected by rain, by generating two other InSAR images for both Kyushu and Shikoku frames (Additional file 1: Figures S1).

For InSAR processing, we use a commercial software package provided by Gamma remote sensing
(Wegmueller et al. 1997). We utilize a $10 \mathrm{~m}$ digital elevation model (DEM) from the Geospatial Information Authority of Japan (GSI) to remove the topographic phase. We use the minimum cost flow algorithm for phase unwrapping (Constantini 1998). During InSAR processing, we apply multi-looking (spatial averaging) 21 looks in range and 28 looks in azimuth for Kyushu, and 24 looks in range and 32 looks in azimuth for Shikoku, which results in the spatial resolutions of $\sim 30 \mathrm{~m}$ for Kyushu and $\sim 34 \mathrm{~m}$ for Shikoku in range and $\sim 52 \mathrm{~m}$ for Kyushu and $\sim 68 \mathrm{~m}$ for Shikoku in azimuth, respectively.

We utilize symmetric SSM to create new sub-bands, each new sub-band with one fifth of the original bandwidth. We create two new sub-band interferograms using the SSM processing strategy similar to Furuya et al. (2017); the details of ALOS-2/PALSAR-2 data sets for both InSAR and SSM processing are shown in Table 1. We employ total multi-looking with 60 looks in range and 88 looks in azimuth for Kyushu, and 93 looks in range and 124 looks in azimuth for Shikoku to accommodate 

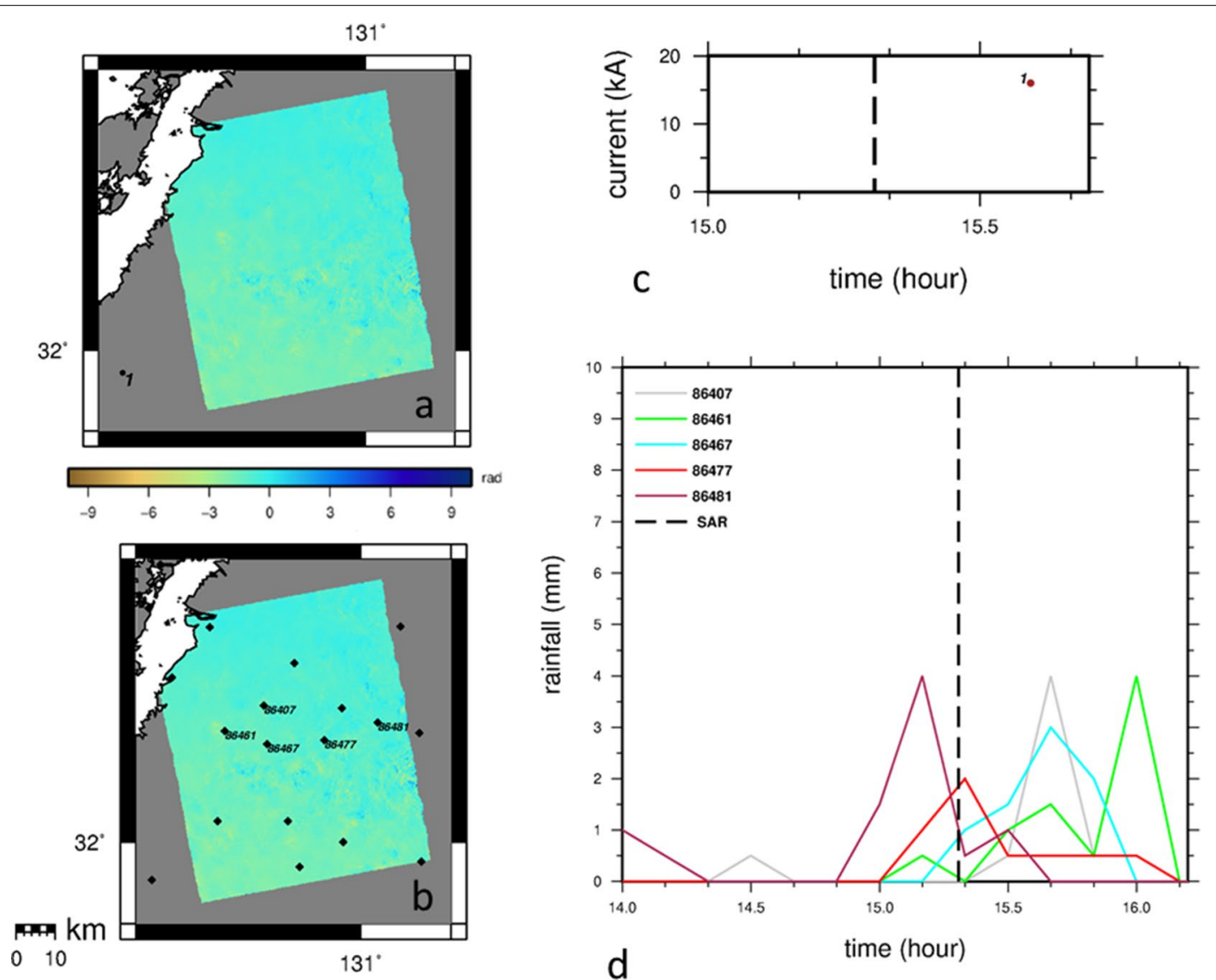

Fig. $5 \Delta \phi_{\text {dispersive }}$ of SSM on Kyushu 20180731_20180828. a Overlaid by lightning location. b Overlaid by the AMeDAS station distribution. The temporal relations of (c) the lightning and (d) 10 min rainfall with the SAR observation time that is indicated with the black dashed line

coarser range resolution derived from the new narrower bandwidth. We do not apply the linear or quadratic plane fitting to mitigate the long-wavelength phase trend to preserve our geophysical interpretation.

\section{InSAR and SSM result}

InSAR heavy rain image of Kyushu shows, in addition to the broad anomaly to the northern edge, three localized anomalies in the middle of the images, each of which increase toward the center and reach $12 \mathrm{~cm}$ along satellite LOS direction (Fig. 2a). Meanwhile, InSAR heavy rain image of Shikoku display an anomaly located near the eastern edge, reaching $16 \mathrm{~cm}$ along satellite LOS direction besides the broad anomalies in the south-west (Fig. 2b). In addition, the coherence map of both InSAR heavy rain images shows a good result (Additional file 1: Figure S2). There is no large surface deformation associated with an earthquake between those acquisition dates of InSAR heavy rain images in Kyushu and Shikoku according to the JMA earthquake catalog.
We do not identify any similar anomalies in fairweather pairs (Additional file 1: Figure S3). As demonstrated previously by InSAR image during rainy conditions, for instance, from the C-band ERS tandem satellite (Hanssen et al. 1999) and L-band ALOS/PALSAR satellite (Kinoshita et al. 2013), these localized anomalies are presumably due to a large water vapor during heavy rain. Next, we apply the SSM to confirm the occurrence of water vapor in the InSAR images in $\Delta \phi_{\text {non-dispersive, }}$ since the water vapor is a non-dispersive medium by definition.

As we set the bandwidth of the SSM to be one-fifth of the original bandwidth, the range resolution is fivetimes coarser, resulting in noisier images. However, the total multi-looking described in the previous sub-section can reduce the increased speckle noise, and the InSAR images with new frequencies (Additional file 1: Figures S4, S5) are quite analogous to the original InSAR images in Fig. 2 and Additional file 1: Figure S3. 


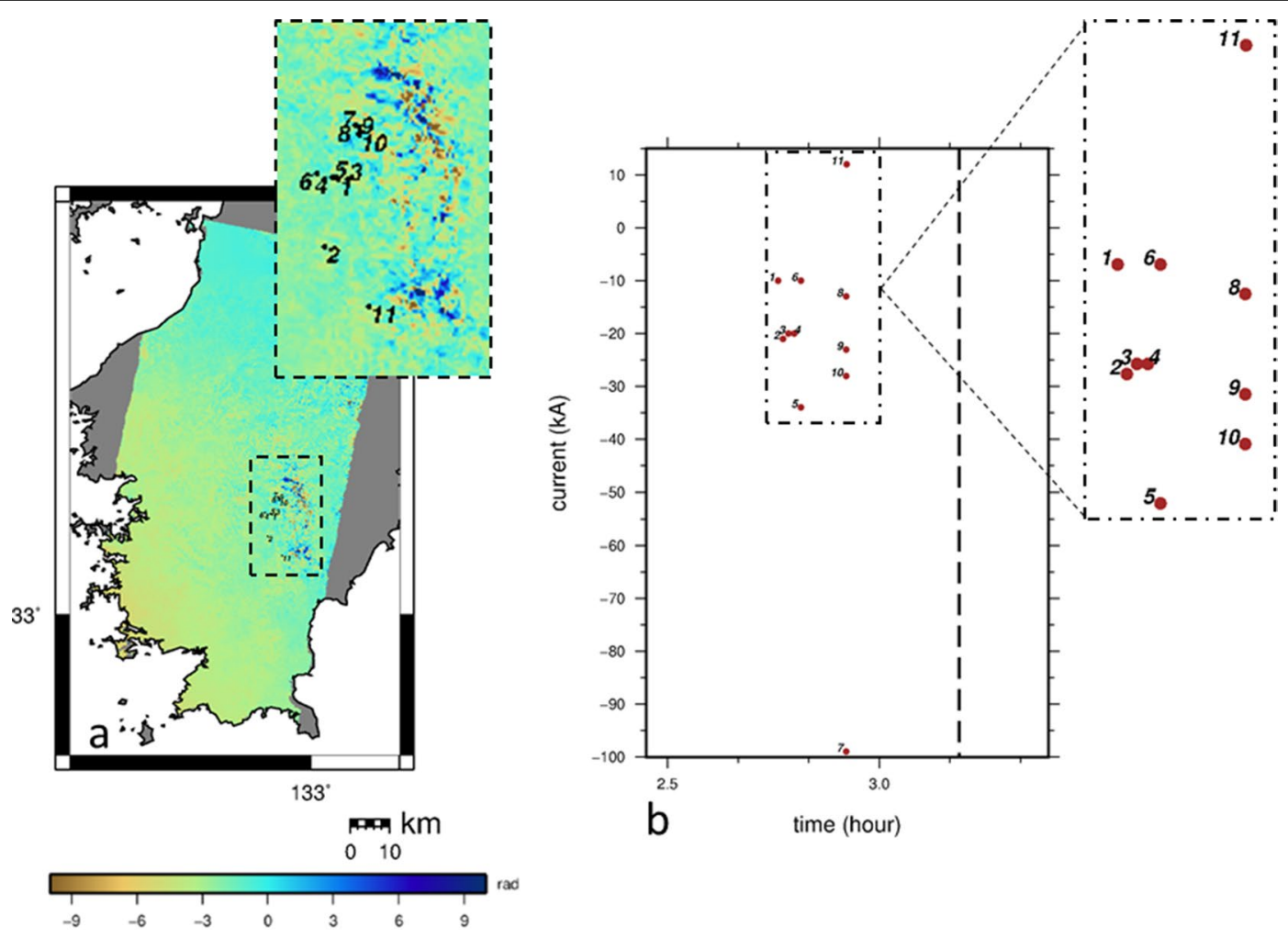

Fig. $6 \Delta \phi_{\text {dispersive }}$ of SSM on Shikoku 20180815_20181107. a Overlaid by lightning location with an expanded view near the lightning. b Temporal relation of the lightning with the SAR imaging time indicated with the black dashed line

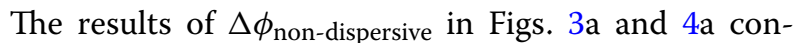
firm the presence of huge water vapor during heavy rain. However, the $\Delta \phi_{\text {dispersive }}$ in Figs. 3b and $4 \mathrm{~b}$ in heavy rain pairs show spotty anomalies aside from the long-wavelength trend induced by the first-order effect of the ionosphere; we confirm the absence of any anomalies both in non-dispersive and dispersive phases in fair weathers (Additional file 1: Figure S7).

\section{Origin of the $\Delta \phi_{\text {dispersive }}$ Spotty anomalies of SSM}

To examine if there were any anomalies in the ionosphere, we check the GNSS ROTI (https://aer-nc-web. nict.go.jp/GPS/GEONET/RMAP/) and the ionosonde data (https://wdc.nict.go.jp/ionog/js_viewer/js_02.html) from the National Institute of Communication Technologies of Japan (NICT). They do not show any anomalies during the heavy rain events, which suggests a steady ionosphere during the SAR acquisitions. We examine the two possible mechanisms originated in the troposphere, by comparing $\Delta \phi_{\text {dispersive }}$ of SSM with ground-based lightning detection network and rain gauge data.
First, we try to associate the spotty signal in $\Delta \phi_{\text {dispersive }}$ of SSM with the runaway electron avalanche during lightning initiation and thundercloud. To confirm our interpretation, we collect lightning data from Japan Lightning Detection Network (JLDN), which tells their location, incident time, electric current, thunder or cloud discharge. The collected lightning data cover about $30 \mathrm{~min}$ before and after the scene centre observation time of SAR data.

In the Kyushu case on 31 July 2018, there is only one lightning occurred $\sim 17$ min after SAR observation time, but this lightning occurred outside the SAR observation scene (Fig. 5a, c). Meanwhile, in the Shikoku data, there occurred a number of lightning, but their occurrences and SAR imaging time are significantly distant, reaching $\sim 15$ min at maximum (Fig. 6a, b). Furthermore, the lightning locations and the unknown spotty signal in the dispersive phase are not matching with each other (Fig. 6a); the lightning clearly occurred on the western side of the spotty signals in $\Delta \phi_{\text {dispersive. }}$. 

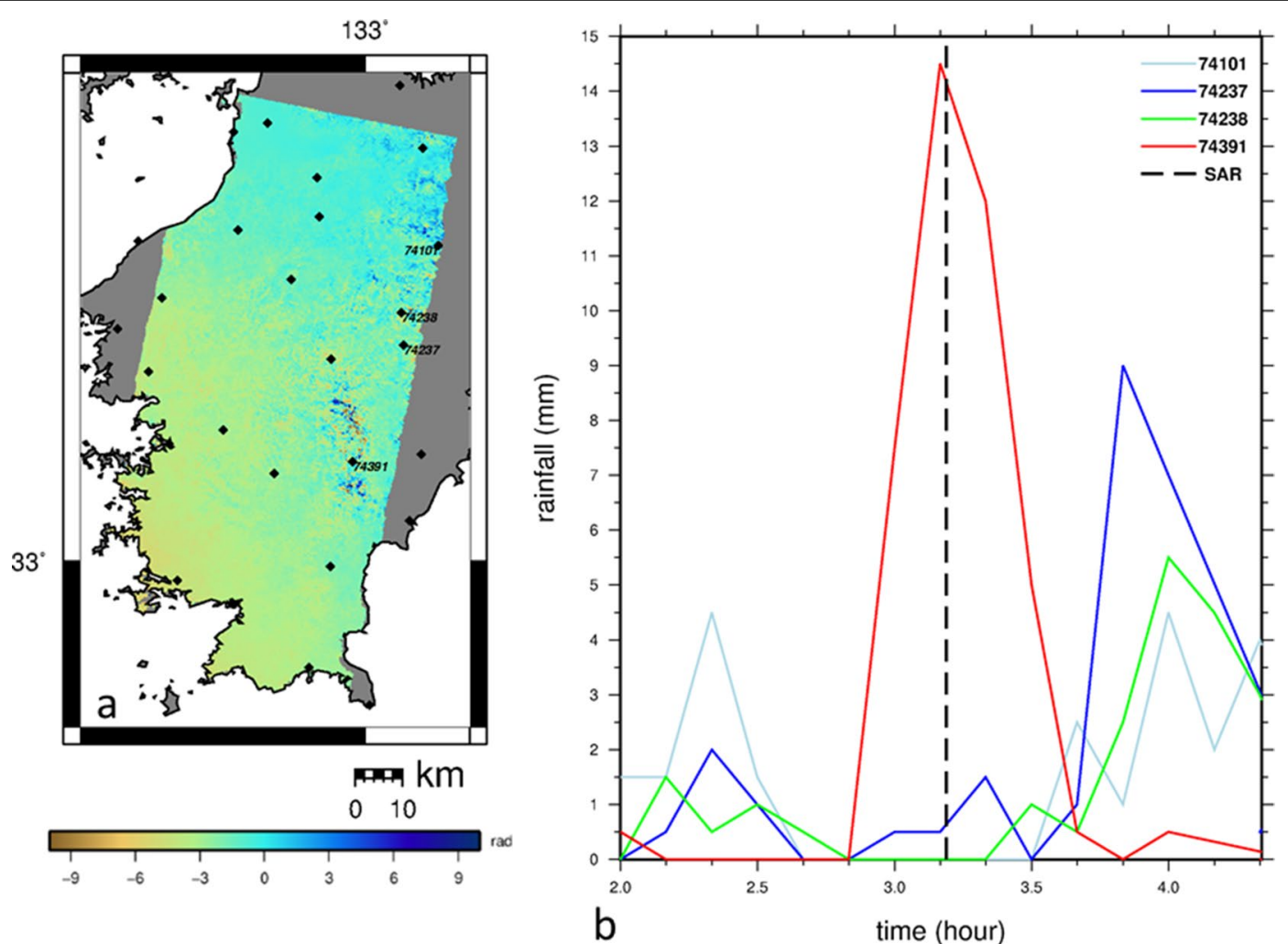

Fig. $7 \Delta \phi_{\text {dispersive }}$ of SSM on Shikoku 20180815_20181107. a Overlaid by the AMeDAS station distribution and (b) the temporal changes in the 10 min rainfall and the SAR imaging time indicated with the black dashed line

Next, we compare $\Delta \phi_{\text {dispersive }}$ with the available rain gauge data derived from AMeDAS, Japanese nationwide ground-based weather observing system, which are distributed with $\sim 17 \mathrm{~km}$ interval and records local rain rate every $10 \mathrm{~min}$. Figures $5 \mathrm{~d}$ and $7 \mathrm{~b}$ show higher rain rate around the SAR imaging time. In particular, the AMeDAS station 74391 located by chance inside the Shikoku heavy rain clearly demonstrates a very high rate by more than $\sim 70 \mathrm{~mm} /$ hour at the time of SAR imaging, supporting that the high rain rate is a likely source for the spotty anomaly in the $\Delta \phi_{\text {dispersive }}$.

The comparison of $\Delta \phi_{\text {dispersive }}$ spotty anomalies with the ground-based lightning detection network and rain gauge data show that the latter rain rate data gives higher correlation in terms of the spatial and temporal matching. We thus prefer an interpretation of the detection of forward scattering by rain droplet to the detection of runaway electron avalanche. However, in contrast to the terrestrial gamma-ray flash that will last only several milliseconds at the time of lightning discharge, another type of gamma-ray emission called "gamma-ray glow" is also known, which can last for several minutes and are not generally accompanied with lightning but are associated with thunderclouds (Wada et al. 2018). We thus consider that few correlations do not necessarily preclude the interpretation of runaway electron avalanche, whereas quantitative assessment of electron content is also necessary.

\section{Presence of another $\Delta \phi_{\text {dispersive }}$ during heavy rain Formulation to detect another dispersive phase}

Given the spotty anomalies in $\Delta \phi_{\text {dispersive }}$ of heavy rain InSAR that presumably originates in the troposphere, we formulate the presence of another dispersive source aside from the first-order free electrons in the ionosphere. Since the SSM only account for phase advance due to the TEC, it is conceivable to add the presence of another dispersive effect in addition to the first order of the ionospheric effect. The new sub-band InSAR images, $\Delta \phi_{\mathrm{H}}$ and $\Delta \phi_{\mathrm{L}}$ will have $\Delta \phi_{\text {another_disp, }}$ aside from $\Delta \phi_{\text {non-dispersive }}$ and $\Delta \phi_{\text {dispersive. }}$ Unlike the conventional $\Delta \phi_{\text {non-dispersive }}$ and the $\Delta \phi_{\text {dispersive }}$ terms, however, no explicit expression 


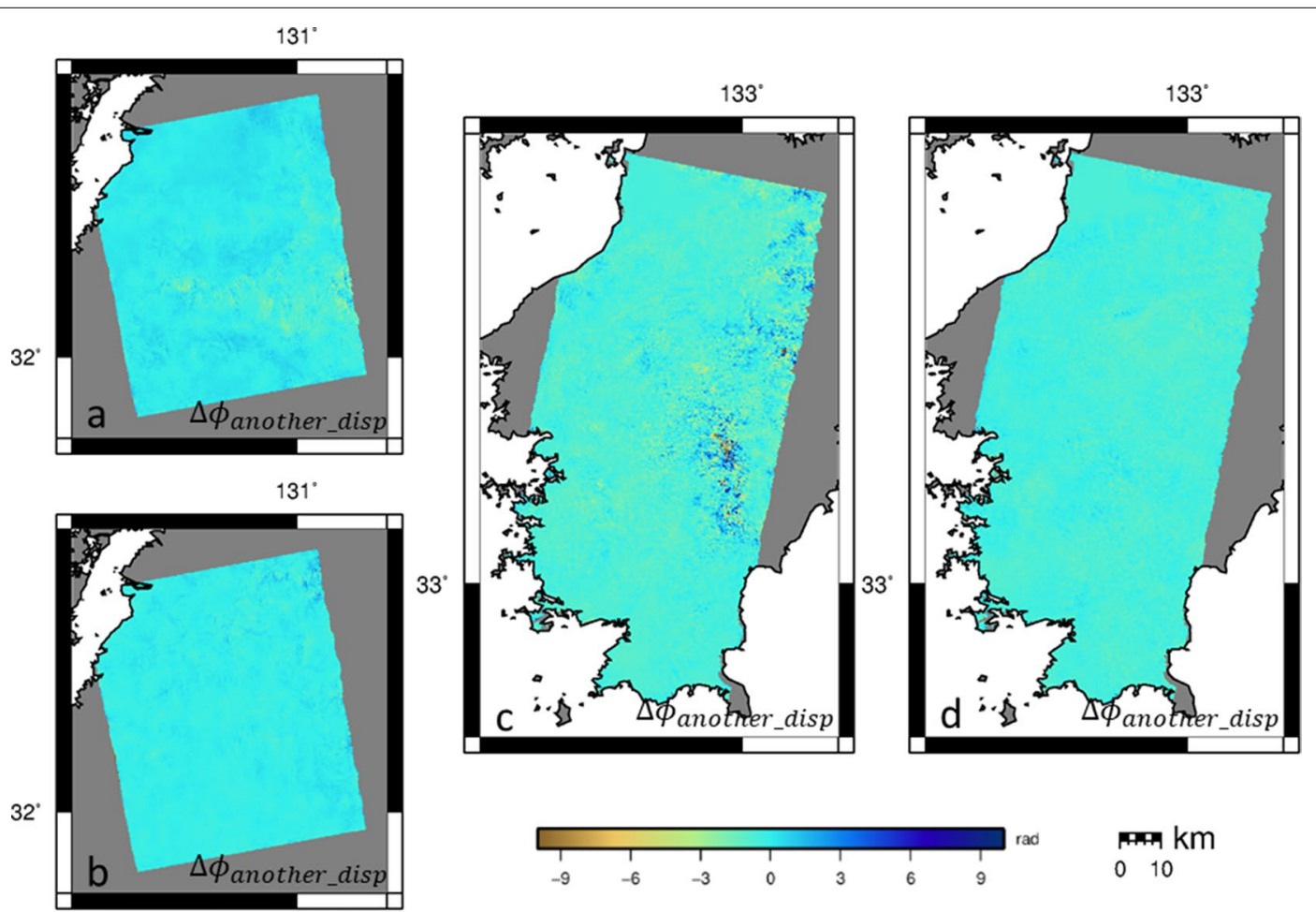

Fig. $8 \Delta \phi_{\text {another disp }}$ derived from triple-frequency SSM. a Kyushu 20180731_20180828 with heavy rain. b Kyushu 20180410_20180828 without heavy rain. c Shikoku 20180815_20181107 with heavy rain. d Shikoku 20180228_20181107 without heavy rain

for its frequency dependence is available, and we thus set it to be $X(f)$ for convenience. To solve the three unknown phases, we need one additional observation, and thus, create the third sub-band InSAR. For simplicity, we use the original center frequency $\Delta \phi_{0}$ with the same reduced bandwidth as $\Delta \phi_{\mathrm{H}}$ and $\Delta \phi_{\mathrm{L}}$ :

$$
\begin{aligned}
\Delta \phi_{\mathrm{H}}= & \Delta \phi_{\text {non-dispersive }} \frac{f_{\mathrm{H}}}{f_{0}}+\Delta \phi_{\text {dispersive }} \frac{f_{0}}{f_{H}} \\
& +\Delta \phi_{\text {another_disp }} X\left(f_{\mathrm{H}}\right) \\
\Delta \phi_{\mathrm{L}}= & \Delta \phi_{\text {non-dispersive }} \frac{f_{\mathrm{L}}}{f_{0}}+\Delta \phi_{\text {dispersive }} \frac{f_{0}}{f_{\mathrm{L}}} \\
& +\Delta \phi_{\text {another_disp }} X\left(f_{\mathrm{L}}\right) \\
\Delta \phi_{0}= & \Delta \phi_{\text {non-dispersive }} \frac{f_{0}}{f_{0}}+\Delta \phi_{\text {dispersive }} \frac{f_{0}}{f_{0}} \\
& +\Delta \phi_{\text {another_disp }} X\left(f_{0}\right)
\end{aligned}
$$

Then, to eliminate $\Delta \phi_{\text {non-dispersive, we divide each new }}$ InSAR sub-band images with each center frequency, followed by subtractions between $\frac{\Delta \phi_{\mathrm{H}}}{f_{\mathrm{H}}}$ and $\frac{\Delta \phi_{\mathrm{L}}}{f_{\mathrm{L}}}$, and between $\frac{\Delta \phi_{0}}{f_{0}}$ and $\frac{\Delta \phi_{\mathrm{L}}}{f_{\mathrm{L}}}$, we get the following:

$$
\begin{aligned}
\frac{\Delta \phi_{\mathrm{H}}}{f_{\mathrm{H}}}-\frac{\Delta \phi_{\mathrm{L}}}{f_{\mathrm{L}}}= & \Delta \phi_{\text {dispersive }}\left(\frac{f_{0}}{f_{\mathrm{H}}^{2}}-\frac{f_{0}}{f_{\mathrm{L}}^{2}}\right) \\
& +\Delta \phi_{\text {another_disp }}\left[X\left(f_{\mathrm{H}}\right) / f_{\mathrm{H}}-X\left(f_{\mathrm{L}}\right) / f_{\mathrm{L}}\right]
\end{aligned}
$$

$$
\begin{aligned}
\frac{\Delta \phi_{0}}{f_{0}}-\frac{\Delta \phi_{\mathrm{L}}}{f_{\mathrm{L}}}= & \Delta \phi_{\text {dispersive }}\left(\frac{f_{0}}{f_{0}^{2}}-\frac{f_{0}}{f_{\mathrm{L}}^{2}}\right) \\
& +\Delta \phi_{\text {another_disp }}\left[X\left(f_{0}\right) / f_{0}-X\left(f_{\mathrm{L}}\right) / f_{\mathrm{L}}\right]
\end{aligned}
$$

Furthermore, we divide Eqs. (4) and (5) with $\left(\frac{1}{f_{\mathrm{H}}^{2}}-\frac{1}{f_{\mathrm{L}}^{2}}\right)$ and $\left(\frac{1}{f_{0}^{2}}-\frac{1}{f_{\mathrm{L}}^{2}}\right)$, respectively, to obtain the following equations:

$$
\begin{aligned}
\frac{\frac{\Delta \phi_{\mathrm{H}}}{f_{\mathrm{H}}}-\frac{\Delta \phi_{\mathrm{L}}}{f_{\mathrm{L}}}}{\left(\frac{1}{f_{\mathrm{H}}^{2}}-\frac{1}{f_{\mathrm{L}}^{2}}\right)}= & \Delta \phi_{\text {dispersive }} f_{0} \\
& +\frac{\Delta \phi_{\text {another_disp }}\left[X\left(f_{\mathrm{H}}\right) / f_{\mathrm{H}}-X\left(f_{\mathrm{L}}\right) / f_{\mathrm{L}}\right]}{\left(\frac{1}{f_{\mathrm{H}}^{2}}-\frac{1}{f_{\mathrm{L}}^{2}}\right)} \\
\equiv & \Gamma\left(f_{\mathrm{H}}, f_{\mathrm{L}}\right)
\end{aligned}
$$



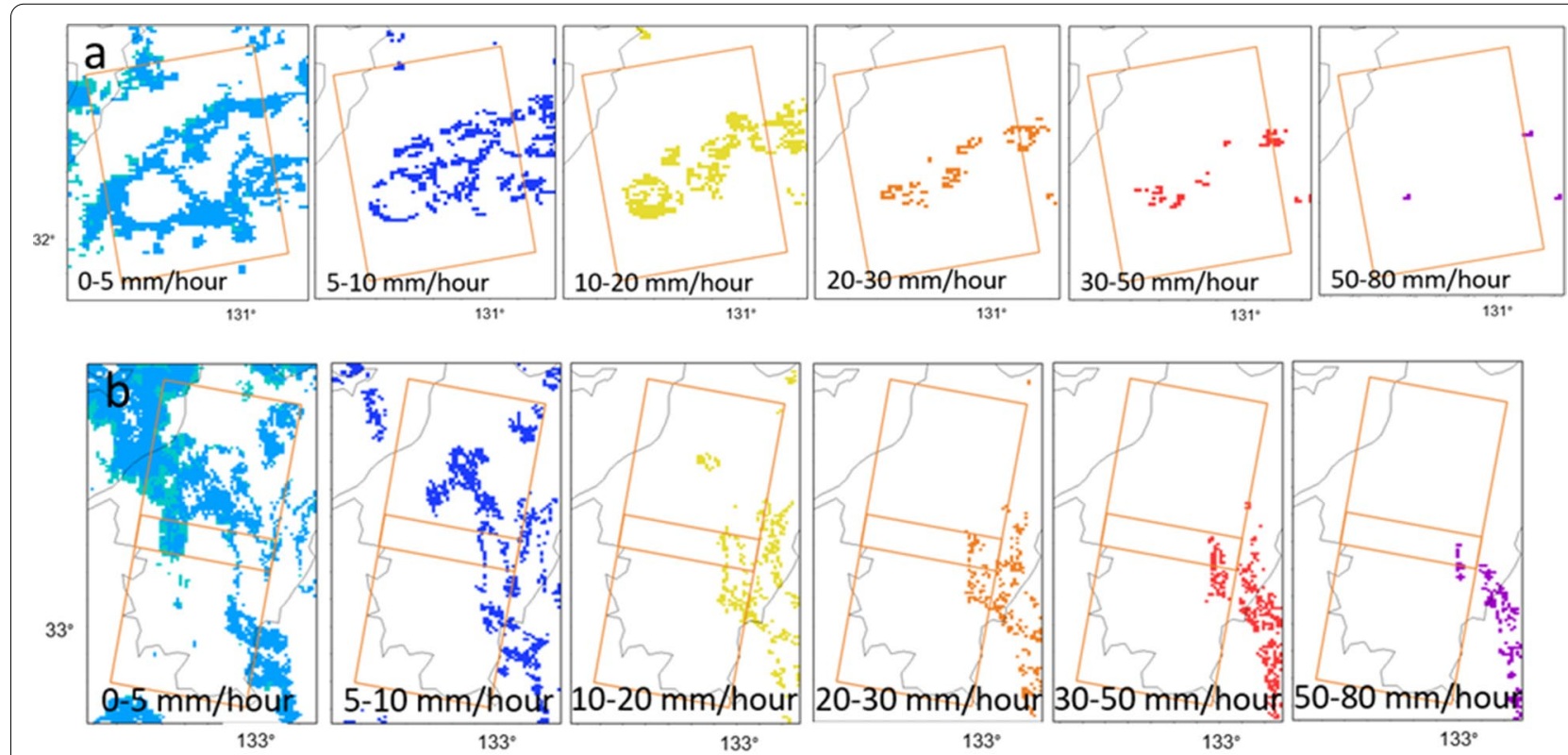

$133^{\circ}$
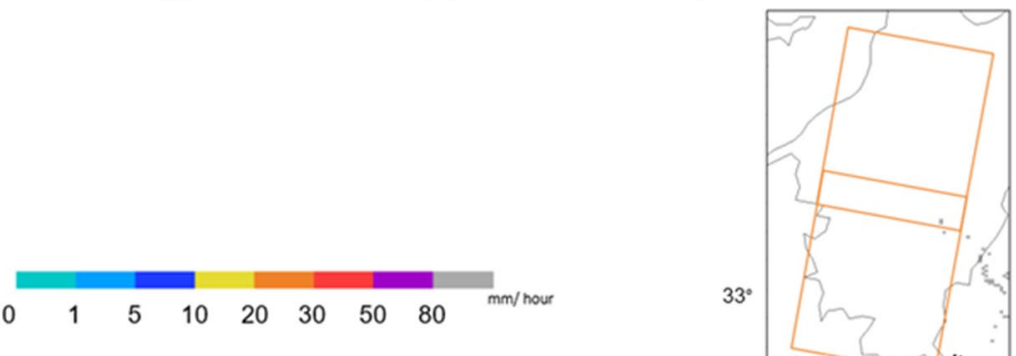

$80-100 \mathrm{~mm} /$ hour

Fig. 9 Rain rate ranges over (a) Kyushu on 20,180,731 at 1520UTC and (b) Shikoku on 20180815 at 0310UTC. Orange squares denote the SAR imaging area taken by ALOS-2/PALSAR-2 SM-1 mode. Colors indicate rain rate in $\mathrm{mm} /$ hour

$$
\begin{aligned}
& \frac{\frac{\Delta \phi_{0}}{f_{0}}-\frac{\Delta \phi_{\mathrm{L}}}{f_{\mathrm{L}}}}{\left(\frac{1}{f_{0}^{2}}-\frac{1}{f_{\mathrm{L}}^{2}}\right)}= \Delta \phi_{\text {dispersive }} f_{0} \\
&+\frac{\Delta \phi_{\text {another_disp }}\left[X\left(f_{0}\right) / f_{0}-X\left(f_{\mathrm{L}}\right) / f_{\mathrm{L}}\right]}{\left(\frac{1}{f_{0}^{2}}-\frac{1}{f_{\mathrm{L}}^{2}}\right)} \\
& \equiv \Gamma\left(f_{0}, f_{\mathrm{L}}\right)
\end{aligned}
$$

We call this function $\Gamma\left(f_{\mathrm{H}}, f_{\mathrm{L}}\right)$ and $\Gamma\left(f_{0}, f_{L}\right)$, which consist of $\Delta \phi_{\text {dispersive }}$ and $\Delta \phi_{\text {another_disp. Subtracting Eq. (7) }}$ from (6) to eliminate $\Delta \phi_{\text {dispersive }} f_{0}$, we can solve for $\Delta \phi_{\text {another_disp }}$ as follows:

$$
\Delta \phi_{\text {another_disp }}=\left[\Gamma\left(f_{\mathrm{H}}, f_{\mathrm{L}}\right)-\Gamma\left(f_{0}, f_{\mathrm{L}}\right)\right] /\left[Y\left(f_{\mathrm{H}}\right)-Y\left(f_{0}\right)\right]
$$

where we replaced $\frac{\left[X\left(f_{\mathrm{H}}\right) / f_{\mathrm{H}}-X\left(f_{\mathrm{L}}\right) / f_{\mathrm{L}}\right]}{\left(\frac{1}{f_{\mathrm{H}}^{2}}-\frac{1}{f_{\mathrm{L}}^{2}}\right)}$ and $\frac{\left[X\left(f_{0}\right) / f_{0}-X\left(f_{\mathrm{L}}\right) / f_{\mathrm{L}}\right]}{\left(\frac{1}{f_{0}^{2}}-\frac{1}{f_{\mathrm{L}}^{2}}\right)}$ with $\left[Y\left(f_{\mathrm{H}}\right)-Y\left(f_{\mathrm{L}}\right)\right]$ and $\left[Y\left(f_{0}\right)-Y\left(f_{\mathrm{L}}\right)\right]$, respectively, for easier notation. In reality, however, the explicit forms of $\mathrm{X}(f)$ and $\mathrm{Y}(f)$ are uncertain. To derive $\Delta \phi_{\text {another_disp con- }}$ sistently with the radian unit, we simply divide the difference, $\Gamma\left(f_{\mathrm{H}}, f_{\mathrm{L}}\right)-\Gamma\left(f_{0}, f_{\mathrm{L}}\right)$, by $10^{9} \mathrm{~Hz}$, so that we can evaluate the magnitude of $\Delta \phi_{\text {another_disp. }}$

\section{Results in heavy rain and fair-weather cases}

First, we create the other $\left(\Delta \phi_{0}\right)$ sub-band images for both heavy rain and fair-weather in Kyushu and Shikoku; we now do not have to shift the center frequency but set the same bandwidth as the other sub-band images. The new images (Additional file 1: Figure S6) are quite similar to 

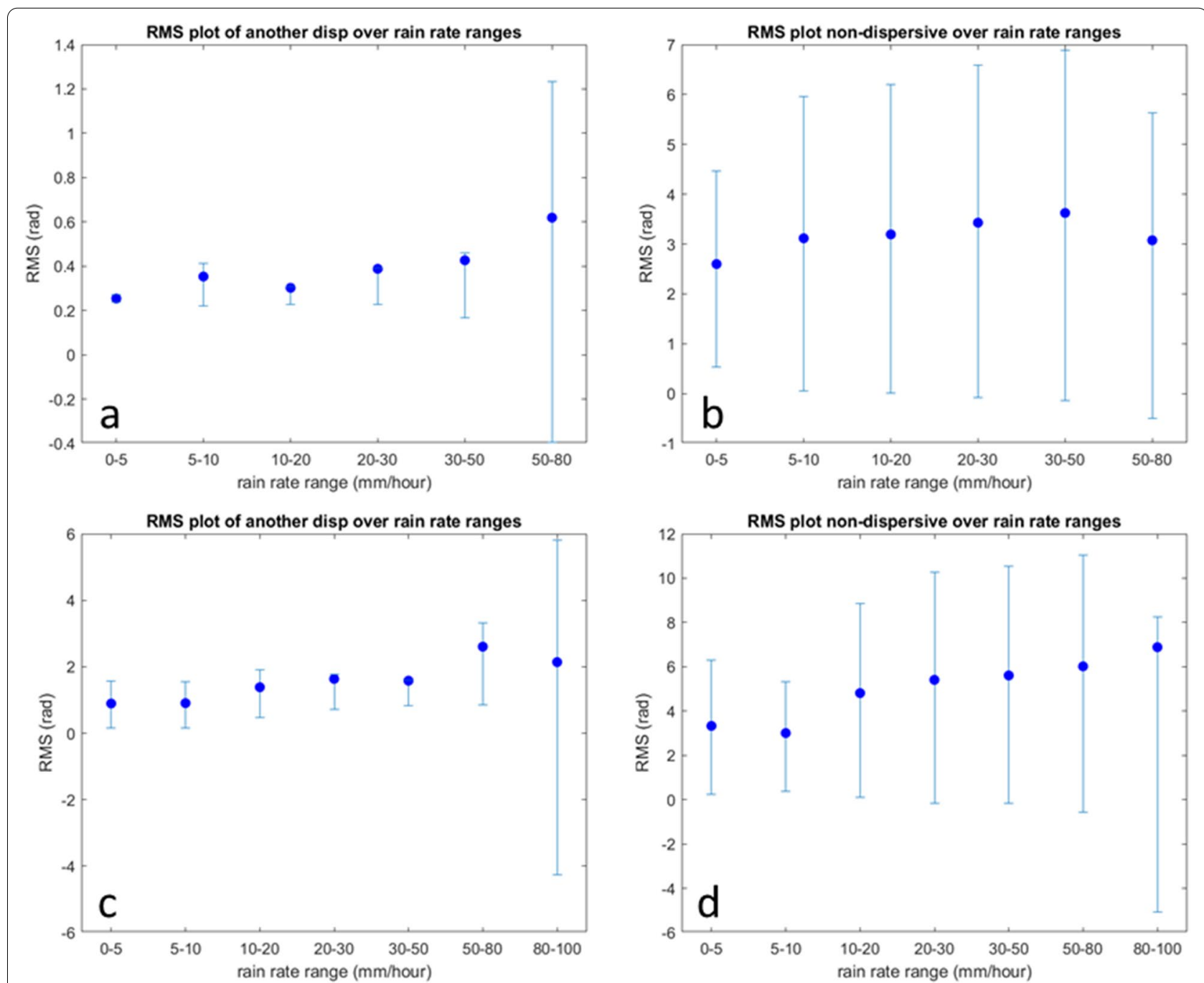

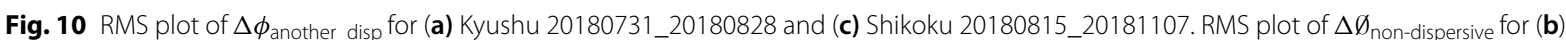
Kyushu 20180731_20180828 and (d) Shikoku 20180815_20181107. The error bars represent 95\% confidence interval

the original InSAR images (Fig. 2; Additional file 1: Figure S2), as well as the previous sub-band SSM images (Additional file 1: Figures S3, S4).

The $\Delta \phi_{\text {another_disp }}$ estimated for both heavy rain and fair-weather cases are shown in Fig. 8. We can clearly observe some spatial phase changes in the heavy rain image (Fig. 8a, c), whereas there are no significant spatial variations in the fair-weather image (Fig. 8b, d). Moreover, compared with the original dispersive phases in Figs. 3b and 4b, we should note that long-wavelength phases are significantly reduced in Fig. 8a, c, suggesting that ionospheric first-order TEC effect could be successfully removed.

\section{Comparison of another dispersive phase with weather radar}

To check if another dispersive phase is related with the local rain rate, we compare the $\Delta \phi_{\text {another_disp }}$ in Fig. $8 \mathrm{a}$, $c$ with various rain rate ranges from the JMA weather radar; the distribution of AMeDAS rain gauge data is too sparse $(\sim 17 \mathrm{~km})$ to compare with the InSAR data. We first categorized the rain captured by JMA weather radar into six and seven rain rate categories for Kyushu and Shikoku, respectively (Fig. 9). Then, we compute the RMS of the $\Delta \phi_{\text {another_disp }}$ at the corresponding rain rate ranges, by selecting a point in weather radar and averaging $\Delta \phi_{\text {another_disp }}$ value in weather radar point vicinity. 


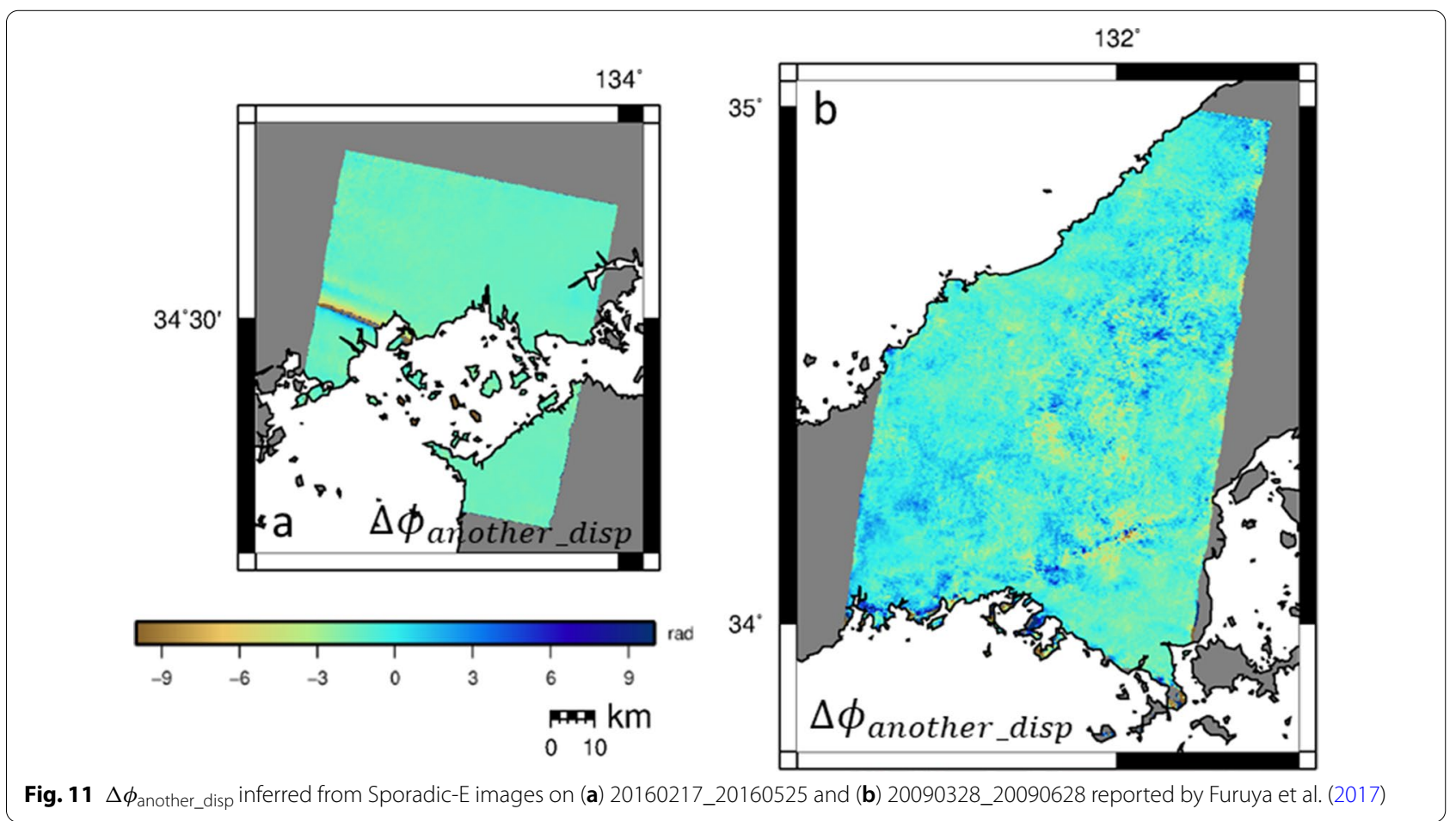

In both Kyushu (Fig. 10a) and Shikoku (Fig. 10c), the RMS values are largely in increasing trend with the rain rate in light of the $95 \%$ confidence intervals, suggesting their positive correlations between them; the large uncertainties at the very high rain rate are due to the small number of sample pixels. As a comparison, we also calcu-

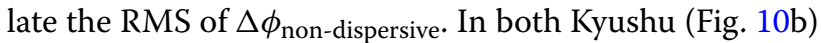
and Shikoku (Fig. 10d), the RMS of $\Delta \phi_{\text {non-dispersive has }}$ larger uncertainties than those in $\Delta \phi_{\text {another_disp, }}$ and there is no clear positive correlation with the rain rate. These contrasting results may verify that the tropospheric dis-

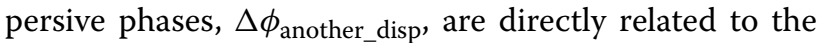
rain rate. As the $\Delta \phi_{\text {non-dispersive is physically attributed to }}$ the water vapor amount, instead of rain itself, the larger uncertainties in $\Delta \phi_{\text {non-dispersive }}$ as well as no positive correlation with rain rate are reasonable.

However, the RMS values in $\Delta \phi_{\text {another_disp }}$ are significantly different between the two locations. This would be because the $\Delta \phi_{\text {another_disp }}$ is simply derived by dividing $\Gamma\left(f_{\mathrm{H}}, f_{\mathrm{L}}\right)-\Gamma\left(f_{0}, f_{\mathrm{L}}\right)$ with $10^{9} \mathrm{~Hz}$ to compare its magnitude with other terms but still includes additional unknown factors that depend not only frequency but also other parameters such as the canting angle of rain drop, local wind direction and so on. In other words, we admit that theoretical modeling to physically relate any meteorological data to tropospheric dispersive phase is still in its infancy, which well deserves further studies.

\section{Confirmation of the higher order ionospheric effect}

To quantitatively confirm the magnitude of another dispersive phase term, we further compute $\Delta \phi_{\text {another_disp }}$ for the Sporadic-E events reported by Furuya et al. (2017) and compare with the heavy rain cases. Furuya et al. (2017) performed minimum-norm inversion to suggest the presence of higher order ionospheric effects without generating the third sub-band InSAR images. In contrast, we have performed triple-frequency measurement by invoking $\Gamma\left(f_{0}, f_{\mathrm{L}}\right)$ in addition to $\Gamma\left(f_{\mathrm{H}}, f_{\mathrm{L}}\right)$ to eliminate both non-dispersive and first order TEC effect. Shown in

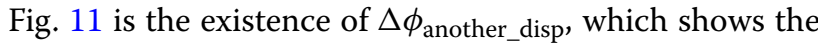
need to account for the higher order ionospheric effect during the Sporadic-E events. Moreover, looking at the $\Delta \phi_{\text {another_disp }}$ from both of heavy rain cases (Fig. 8a, c) and Sporadic-E (Fig. 11), we may conclude that the tropospheric dispersive phase in heavy rain seems to have the similar order of magnitude in comparison to the higher dispersive phase in the ionosphere during Sporadic-E events.

\section{Conclusion}

We generated InSAR images that captured two heavy rain episodes in Japan and calculated $\Delta \phi_{\text {non-dispersive }}$ and $\Delta \phi_{\text {dispersive }}$ using the SSM. While $\Delta \phi_{\text {non-dispersive }}$ is dominated presumably by the large water vapor signals, there are spotty anomalies in the $\Delta \phi_{\text {dispersive }}$ in the rainy area. 
We proposed two distinct physical mechanisms and examined them with ground-based lightning observation and rain gauge data. As a result, the rain scattering dispersive effect is spatiotemporally more preferable to the runaway electron avalanche, demonstrating the possibility of simultaneous observation of rain droplet and water vapor with a single instrument at the same high spatial resolution. Based on a formulation to include another dispersive effect, we confirmed that another dispersive effect does exist during heavy rain but is absent in fair weather. In addition, the $\Delta \phi_{\text {another_disp }}$ originated probably from rain-droplet show a positive correlation with rain radar data, whereas the water-vapor driven $\Delta \phi_{\text {non-dispersive }}$ does not. Another dispersive phase associated with heavy rain is likely to have the same order of magnitude to the higher order ionospheric effect during sporadic-E.

\section{Abbreviations}

AMeDAS: Automated meteorological data acquisition system; ALOS-2: Advanced land observation satellite-2; DEM: Digital elevation model; GNSS: Global Navigation Satellite System; GSI: Geospatial Information Authority of Japan; InSAR: Interferometric synthetic aperture radar; JLDN: Japan Lightning Detection Network; JMA: Japan Meteorological Agency; LOS: Line of sight; MCF: Minimum cost flow; NICT: National Institute of Communications and Technology of Japan; PALSAR-2: Phased array L-band synthetic aperture radar-2; RMS: Root mean square; ROTI: Rate of total electron content index; SAR: Synthetic aperture radar; SM-1: Strip map mode-1; SSM: Split-spectrum method; TEC: Total electron content.

\section{Supplementary Information}

The online version contains supplementary material available at https://doi. org/10.1186/s40623-021-01470-9.

Additional file 1: Figure S1. JMA weather radar observation; on Kyushu at (a) 20180410_1520 UTC, (b) 20180828_1520UTC and Shikoku at (c) 20180228_0310UTC, (d) 20181107_0310UTC. Orange square denote the SAR imaging area taken by ALOS-2/PALSAR-2 SM-1 mode. Figure S2. Coherence map of original bandwidth differential interferogram of heavy rain pairs; (a) Kyushu on 20180731_20180828, and (b) Shikoku 20180815_20181107. Figure S3. Original bandwidth differential interferogram of fair-weather pairs; (a) Kyushu on 20180410_20180828, and (b) Shikoku 20180228_20181107. Figure S4. Unwrapped differential interferogram for heavy rain pairs. Kyushu 20180731_20180828 with (a) high frequency, (b) low frequency and Shikoku 20180815_20181107 with (c) high frequency, (d) low frequency. Figure S5. Unwrapped differential interferogram for fair-weather pairs. Kyushu 20180731_20180828 with (a) high frequency, (b) low frequency and Shikoku 20180815_20181107 with (c) high frequency, (d) low frequency. Figure S6. The retrieval of standard SSM on Kyushu 20180731_20180828; (a) $\Delta \phi_{\text {non-dispersive, }}$ and (b) $\Delta \phi_{\text {dispersive; }}$ and Shikoku $20180228 \_20181107$ (c) $\Delta \phi_{\text {non-dispersive, }}$ and (d) $\Delta \phi_{\text {dispersive. }}$ Figure S7. Unwrapped differential interferogram of F0; (a) Kyushu 20180731_20180828 (b) Kyushu 20180410_20180828, (c) Shikoku 20180815_20181107,(d) Shikoku 20180228_20181107.

\section{Acknowledgements}

The ALOS-2/PALSAR-2 level 1.1 data in this study are shared among PIXEL (PALSAR Interferometry Consortium to Study our Evolving Land Surface) under a cooperative research contract with the Earthquake Research Institute, University of Tokyo. The ownership of ALOS-2/PALSAR-2 data belongs to JAXA. The ownership of weather radar and AMeDAS data belongs to the JMA. We acknowledge two anonymous reviewers and an editor whose comments were helpful to revise the original manuscript.

\section{Authors' contributions}

MF conceptualize the research and supervise during the research. NS search and process heavy rain cases. Both of the authors write, read, and approved the final manuscript.

\section{Funding}

NS is grateful to JICA Innovative Asia for financially supporting his master's study at Hokkaido University. This study also partially supported by Specific Research Project (B), "Study on the crustal and surface deformation using multiple SAR techniques, at Earthquake Research Institute, the University of Tokyo.

\section{Availability of data and materials}

The ALOS-2/PALSAR-2 level 1.1 used in this study available upon purchase through RESTEC (https://www.restec.or.jp/en/) or PASCO (http://en.alos-pasco. com). We can share the data upon request within a framework of collaboration with our research group. We used the GAMMA (https://www.gamma-rs. ch), and MATLAB for processing. The weather radar and AMeDAS data are accessible and the courtesy of the Japan Meteorological Agency. The weather data also accessible from (http://database.rish.kyoto-u.ac.jp/arch/jmadata/ data/jma-radar/synthetic/original/) maintained by RISH, Kyoto University. The lightning data available upon purchase from Franklin Japan (https://www. franklinjapan.jp/).

\section{Declarations}

Ethics approval and consent to participate

Not applicable.

\section{Competing interests}

The authors declare that they have no competing interests.

\section{Author details}

${ }^{1}$ Department of Natural History Sciences, Graduate School of Science, Hokkaido University, Sapporo, Japan. ${ }^{2}$ Department of Earth and Planetary Sciences, Faculty of Science, Hokkaido University, Sapporo, Japan.

Received: 24 February 2021 Accepted: 23 June 2021

Published online: 16 July 2021

\section{References}

Belcher DP (2008) Theoretical limits on SAR imposed by the ionosphere. IET Radar Sonar Navig 2(6):435-448. https://doi.org/10.1049/iet-rsn:20070188 Bevis M, Businger S, Chiswell S, Herring TA, Anthes RA, Rocken C, Ware RH (1994) GPS meteorology: mapping zenith wet delays onto precipitable water. J Appl Meteorol. 33(3): 379-386. https://doi.org/10.1175/15200450(1994)033<0379:gmmzwd>2.0.co;2

Brcic R, Parizzi A, Eineder M, Bamler R, Meyer F (2010) Estimation and compensation of ionospheric delay for SAR interferometry. IEEE Int Geosci Remote Sens Symp. https://doi.org/10.1109/igarss.2010.5652231

Costantini M (1998) A Novel Phase Unwrapping Method Based on Network Programming. IEEE Trans Geosci Remote Sens 36(3):813-821. https://doi. org/10.1109/36.673674

Crane RK (1967) Coherent pulse transmission through rain. IEEE Trans Antennas Propag 15(2):252-256. https://doi.org/10.1109/tap.1967.1138897

Curlander JC, McDonough RN (1991) Synthetic aperture radar: systems and signal processing. Wiley

Dwyer JR (2003) A fundamental limit on electric fields in air. Geophys Res Lett 30:2055. https://doi.org/10.1029/2003GL017781

Dwyer JR, Smith DM, Cummer SA (2012) High-energy atmospheric physics: terrestrial gamma-ray flashes and related phenomena. Space Sci Rev 173(1-4):133-196. https://doi.org/10.1007/s11214-012-9894-0 
Furuya M, Suzuki T, Maeda J, Heki K (2017) Midlatitude sporadic-E episodes viewed by L-band split-spectrum InSAR. Earth, Planets Space 69(1). https://doi.org/10.1186/s40623-017-0764-6

Gomba G, Parizzi A, Zan FD, Eineder M, Bamler R (2016) Toward operational compensation of ionospheric effects in SAR interferograms: the splitspectrum method. IEEE Trans Geosci Remote Sens 54(3):1446-1461. https://doi.org/10.1109/tgrs.2015.2481079

Gurevich AV, Milikh GM, Roussel-Dupre R (1992) Runaway electron mechanism of air breakdown and preconditioning during a thunderstorm. Phys Lett A 165(5-6):463-468. https://doi.org/10.1016/0375-9601(92)90348-p

Gurevich AV, Medvedev YV, Zybin KP (2004) New type discharge generated in thunderclouds by joint action of runaway breakdown and extensive atmospheric shower. Phys Lett A 329(4-5):348-361. https://doi.org/10. 1016/j.physleta.2004.06.099

Hanssen RF, Weckwerth TM, Zebker HA, Klees R (1999) High-resolution water vapor mapping from interferometric radar measurements. Science 283(5406):1297-1299. https://doi.org/10.1126/science.283.5406.1297

Hanssen RF (2001) Radar interferometry: data interpretation and error analysis. Kluwer Academic Publishing

Hobiger T, Jakowski N (2017) Atmospheric signal propagation. In: Teunissen PJG, Montenbruck O (eds) Springer handbook of global navigation satellite systems. Springer, Berlin, pp 165-193

Jung HS, Lee DT, Lu Z, Wong JS (2013) lonospheric correction of SAR interferograms by multiple-aperture interferometry. IEEE Trans Geosci Remote Sens 51(5):3191-3199. https://doi.org/10.1109/tgrs.2012.2218660

Kedar S, Hajj GA, Wilson BD, Heflin MB (2003) The effect of the second order GPS ionospheric correction on receiver positions. Geophys Res Lett 30(16). https://doi.org/10.1029/2003gl017639

Kelley NA, Smith DM, Dwyer JR, Splitt M, Lazarus S, Martinez-Mckinney F, Hazelton B, Grefenstette B, Lowell A, Rassoul HK (2015) Relativistic electron avalanches as a thunderstorm discharge competing with lightning. Nat Commun 6(1). https://doi.org/10.1038/ncomms8845

Kinoshita Y, Shimada M, Furuya M (2013) InSAR observation and numerical modeling of the water vapor signal during a heavy rain: a case study of the 2008 Seino Event, Central Japan. Geophys Res Lett 40(17):4740-4744. https://doi.org/10.1002/grl.50891

Liebe HJ (1989) MPM — an atmospheric millimeter-wave propagation model. Int J Infrared Milli Waves 10:631-650. https://doi.org/10.1007/BF01009565

Meyer F, Bamler R, Jakowski N (2006) The potential of low-frequency SAR systems for mapping ionospheric TEC distributions. IEEE Geosci Remote Sens Lett 3(4):560-564. https://doi.org/10.1109/lgrs.2006.882148
Oguchi T (1983) Electromagnetic wave propagation and scattering in rain and other hydrometeors. Proc IEEE 71(9):1029-1078. https://doi.org/10.1109/ PROC.1983.12724

Rosen PA, Hensley S, and Chen C (2010) Measurement and mitigation of the ionosphere in L-band interferometric SAR Data. 2010 IEEE Radar Conference. https://doi.org/10.1109/radar.2010.5494385

Saito A, Fukao S, Miyazaki S (1998) High resolution mapping of TEC perturbations with the GSI GPS network over Japan. Geophys Res Lett 25(16):3079-3082. https://doi.org/10.1029/98gl52361

Shoji Y (2013) Retrieval of water vapor inhomogeneity using the Japanese nationwide GPS array and its potential for prediction of convective precipitation. J Meteorol Soc Japan Ser II. 91(1):43-62. https://doi.org/10. 2151/jmsj.2013-103

Solheim FS, Vivekanandan J, Ware RH, Rocken C (1999) Propagation delays induced in GPS signals by dry air, water vapor, hydrometeors, and other particulates. J Geophys Res Atmos 104(D8):9663-9670. https://doi.org/10. 1029/1999jd900095

Thayer GD (1974) An improved equation for the radio refractive index of air. Radio Sci 9(10):803-807. https://doi.org/10.1029/rs009i010p00803

van de Hulst HC (1957) Light scattering by small particles. Wiley

Wada Y, Bowers GS, Enoto T, Kamogawa M, Nakamura Y, Morimoto T, Smith DM, Furuta Y, Nakazawa K, Yuasa T, Matsuki A, Kubo M, Tamagawa T, Makishima K, Tsuchiya H (2018) Termination of electron acceleration in thundercloud by intracloud/intercloud discharge. Geophys Res Lett 45(11):5700-5707. https://doi.org/10.1029/2018gl077784

Wegmüller U, Werner CL (1997) GAMMA SAR processor and interferometry Software, Proceedings 3rd ERS Scientific Symposium, Florence, Italy, 17-20 March 1997

Wessel P, Luis JF, Uieda L, Scharroo R, Wobbe F, Smith WHF, Tian D (2019) The generic mapping tools version 6. Geochem Geophys Geosyst 20:55565564. https://doi.org/10.1029/2019GC008515

Zufferey $\mathrm{CH}$ (1972) A study of rain effects on electromagnetic waves in the 1-600 GHZ Range. University of Colorado

\section{Publisher's Note}

Springer Nature remains neutral with regard to jurisdictional claims in published maps and institutional affiliations.

\section{Submit your manuscript to a SpringerOpen ${ }^{\circ}$ journal and benefit from:}

- Convenient online submission

- Rigorous peer review

- Open access: articles freely available online

- High visibility within the field

- Retaining the copyright to your article

Submit your next manuscript at $\boldsymbol{\nabla}$ springeropen.com 